\title{
Biosensing Based on Light Absorption of Nanoscaled Gold and Silver Particles
}

\author{
Filip Frederix, ${ }^{*, \dagger} \mathrm{J}$ ean-Mic hel Friedt, ${ }^{\dagger}$ Kang-Hoon Choi, ${ }^{\dagger}$ Wim Laureyn, ${ }^{\dagger}$ Andrew Campitelli, ${ }^{\dagger}$ \\ Dirk Mondelaers, ${ }^{\ddagger}$ Guido Maes, ${ }^{\S}$ and Gustaaf Borghs ${ }^{\dagger}$ \\ MCP-BIO, IMEC vzw, Kapeldreef 75, B-3001 Leuven, Belgium, Laboratory of Inorganic and Physical Chemistry, \\ Limburgs Universitair Centrum, IMO, B-3590 Diepenbeek, Belgium, and Chemistry Department, KULeuven, \\ Celestijnenlaan 200 F, B-3001 Leuven, Belgium
}

The absorption spectrum of noble metal spherical nanoparticles is known to be strongly influenced by the dielectric constant of the surrounding material, and as such, these particles are well suited for biosensing applications. To perform biosensing using nanoparticles on a substrate, the metal particles are covalently attached onto quartz using an organic adhesion layer of mercaptosilanes. The particles in solution are characterized by UV-vis spectroscopy and transmission electron microscopy, while those attached to the quart are characterized with UV-vis spectroscopy and atomic force microscopy. Antibodies are attached to the metal nanoparticles, and the antigen recognition is monitored via the change of light absorption when this binding event occurs. Not only is the absorbance originating from plasmon resonances of the particles influenced by the dielectric properties of molecules attached to the nanospheres but also the interband absorption of the particles changes, which will be demonstrated in this report. A light absorption change is detected when a molecular recognition occurs between the bioreceptor molecules attached to the nanoparticle and a biomolecular counterpart. This change in absorption can be very large when adhered molecules are at resonance (interband transitions). In addition, the presented type of biosensing can be a cost-effective and easy to use alternative to conventional biosensing techniques.

Surface plasmon resonance (SPR) sensors are widely used for biosensing, especially as affinity biosensors. ${ }^{1,2}$ Such devices are based on the high sensitivity of the surface plasmon resonance of a thin metal film to refractive index changes when a coating is applied onto the metal surface. ${ }^{3}$ The high sensitivity of SPR is due to the large enhancement of the electromagnetic field at the interface. Plasmon resonance absorption of particles has been used to investigate the streptavidin-biotin interaction on silver particles

* To whom correspondence should be addressed. E-mail: Frederix@ imec.be.

† IMEC vzW.

₹ Limburgs Universitair Centrum.

$\S$ KULeuven.

(1) Cooper, M. A. Nat. Rev. 2002, 1, 515.

(2) Rogers, K. R. Mol. Biotechnol. 2000, 14, 109.

(3) Raether, H. Surface Plasmons on Smooth and Rough Surfaces and on Gratings; Springer-Verlag: Berlin, 1998. deposited on glass and to investigate thiol adsorption on gold particles. ${ }^{4,5} \mathrm{It}$ is demonstrated in this report that particle plasmon resonance as well as the bulk absorption of small gold and silver particles is sensitive to adsorbed interface layers and it is therefore very promising to be used for biosensing. In some cases the interband absorbance of the particles attached to quartz is even more sensitive to changes in the dielectric properties upon binding of biomolecules, compared to plasmon resonance absorbance changes. This remarkable and novel phenomenon will be elucidated in this report.

The absorption spectrum of noble metal nanoparticles shows both bulk interband absorption and particle plasmon resonances. The latter are collective oscillations of the conduction electrons on the surface of the small particle. The resonance frequency of this particle plasmon is determined mainly by the dielectric functions of the metal and the surrounding medium and by the particle shape, i.e. the ratio of the principal axes. ${ }^{6}$ Resonances lead to a narrow spectrally selective absorption and to an enhancement of the local light field confined on and close to the surface of the metal particle. 7,8 The surrounding medium influences both the plasmon frequency and the amplitude of the absorption. The interband absorption on the other hand involves the promotion of an electron from the occupied d-level state in the noble metal to an empty state above the Fermi level. This absorption is strongly determined by the joint density of $d$ and $s$ states of the conduction electrons and occurs at shorter wavelengths. Due to the large skin-depth, the nanoparticles absorb light in the whole bulk area of the particle. By increase of the dielectric constant near the nanoparticle surface, for example by biomolecular adsorption, an increase of the density of the electromagnetic field at the particles position (basically a Lorentz local field correction) enlarges the transition probability and as such the absorption for bulk transitions. This effect is only visible when the particle is smaller than the wavelength of the impinging light because this object has a too small lateral extent to support any

(4) Haes, A. J.; Van Duyne, R. P. J. Am. Chem. Soc. 2002, 124, 10596.

(5) Kalyuzhny, G.; Vaskevich, A.; Schneeweiss, M. A.; Rubinstein, I. Chem.Eur. J. 2002, 8 (17), 3849.

(6) Kreibig, U.; Vollmer, M . Optical Properties of M etal Clusters; Springer Series in M aterials Science, Vol. 25; Springer: Berlin, 1995.

(7) Sönnichsen, C.; Geier, S.; Hecker, N. E.; von Plessen, G.; Feldmann, J.; Ditlbacher, H.; Lamprecht, B.; Krenn, J. R.; Aussenegg, F. R.; Chan, V. Z.H.; Spatz, J. P.; M öller, M. Appl. Phys. Lett. 2000, 77, 2949.

(8) Lamprecht, B.; Krenn, J. R.; Leitner, A.; Aussenegg F. R. Phys. Rev. Lett. 1999, 83, 4421.

10.1021/ac0346609 CCC: $\$ 25.00$ @ 2003 American Chemical Society Published on Web 11/07/2003 
(a)

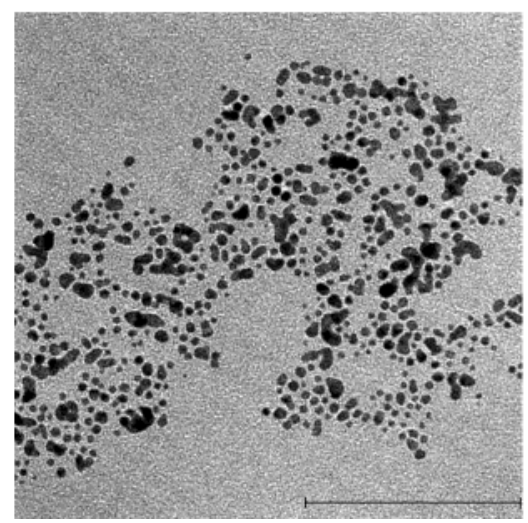

(b)

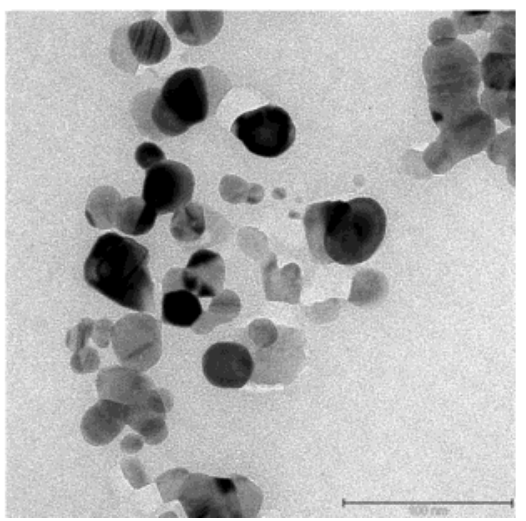

Figure 1. (a) Transmission electron microscope image of gold nanoparticles. The scale bar represents $100 \mathrm{~nm}$. (b) Transmission electron microscope image of silver nanoparticles. The scale bar represents $100 \mathrm{~nm}$.
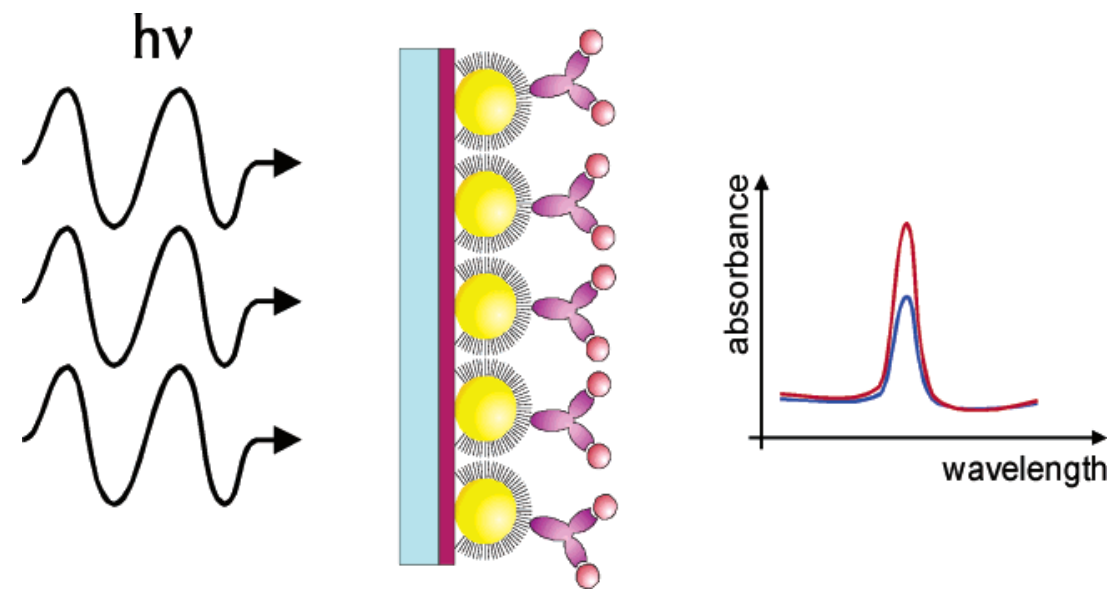

Figure 2. Schematic representation of the applied biosensing principle based on gold and silver nanoparticles deposited on a quartz substrate. From left to right: light source; quartz substrate; mercaptosilane adhesion layer; gold or silver nanoparticles; self-assembled monolayer of functional thiols; antibodies and antigens. The resulting absorbance spectra increase upon binding of analytes to the nanoparticles is shown on the right-hand side of the figure.

purely internal optical mode. The electric field operator internal to such a sphere is determined by the extended modes and hence by the dielectric constant of the surroundings. ${ }^{9}$ At molecular resonances, a strong enhancement of absorption is observed. As the dielectric constant increases, an increased absorption is expected and experimentally verified. This may be different for particle plasmons because the dielectric constant of the surroundings also has a strong influence on the wavenumber and on the strength of the collective and evanescent modes of excitation. If the particles are coated with a different material, both a shift in frequency and in absorption should be observed.

\section{EXPERIMENTAL SECTION}

Materials. 16-M ercapto-1-hexadecanoic acid (16-M HA) and hydrogen tetrachloroaurate $\left(\mathrm{HAuCl}_{4} \cdot 3 \mathrm{H}_{2} \mathrm{O}\right)$ from Aldrich and silver nitrate $\left(\mathrm{AgNO}_{3}\right)$ from VWR were used as received. $\mathrm{Hy}$ droxylamine monohydrochloride was supplied by Fluka, and (3mercaptopropyl)methyltriethoxysilane, by ABCR. Ultrapure methanol, ethanol, and $2 \mathrm{M} \mathrm{NaOH}$ were purchased from Riedel-D eHäen,

(9) Yablonovitch, E.; Gmitter, T. C.; Bhat, R. Phys. Rev. Lett. 1988, 61, 2546. while $\mathrm{HCl}(37 \% \mathrm{v} / \mathrm{v}), \mathrm{HNO}_{3}(70 \% \mathrm{v} / \mathrm{v}), \mathrm{H}_{2} \mathrm{O}_{2}(30 \% \mathrm{v} / \mathrm{v})$, and $\mathrm{NH}_{4}-$ $\mathrm{OH}(25 \% \mathrm{v} / \mathrm{v})$ were purchased from Ashland Chemicals. Human serum albumin (HSA), human IgG reagent (I2511), and sodium borohydride were purchased from Sigma, and anti-human serum albumin (anti-HSA), from Dako. Phosphate-buffered saline (PBS) consists of $10 \mathrm{mM} \mathrm{KH_{2 }} \mathrm{PO}_{4}$ (Sigma) and $\mathrm{Na}_{2} \mathrm{HPO}_{4}$ (Sigma) and $150 \mathrm{mM} \mathrm{NaCl}$ (Calbiochem). Quartz slides were purchased at Chintele Quartz Technology Co.

Instrumentation. UV-vis spectroscopic studies were carried out using a Shimadzu UV-1601PC with a slit width of $2 \mathrm{~nm}$ and a data interval of $0.5 \mathrm{~nm}$. The gold and silver particle coated substrates were measured in air by placing the slides perpendicular to the light beam. The characterization of these slides in solution was performed by placing the slides in the middle of a modified quartz cell. Mixing of the solutions is not performed, which makes this setup very sensitive to mass transport limitations. Atomic force microscopy (AFM) surface images were acquired in tapping mode under ambient conditions (PicoSPM , Molecular Imaging, Tempe, AZ). Si cantilevers having a spring constant between 1.2 and $5.5 \mathrm{~N} / \mathrm{m}$ were used at resonance frequencies between 60 and $90 \mathrm{kHz}$. Transmission electron 


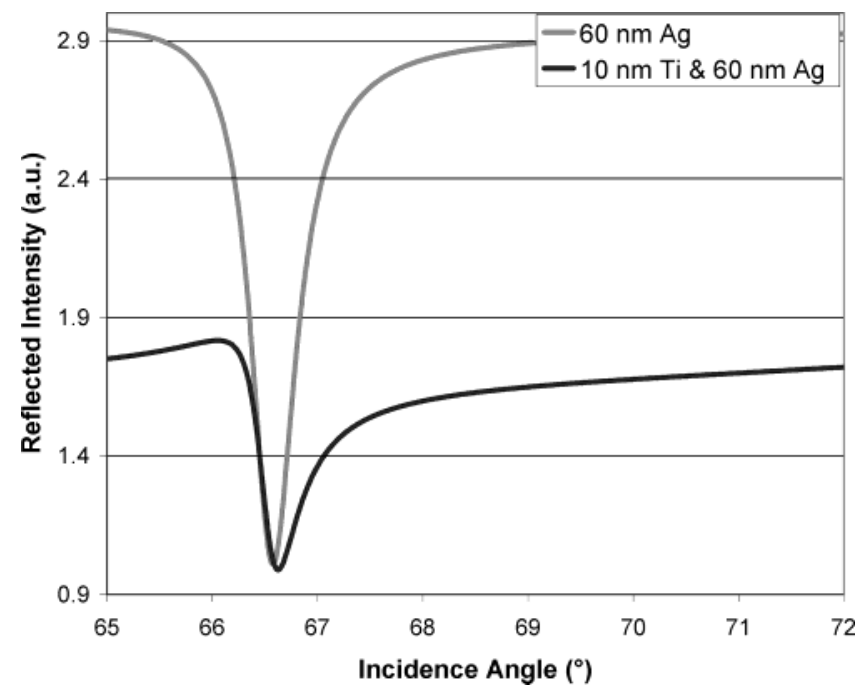

Figure 3. Simulation of the plasmon resonance peaks of silver with and without a $\mathrm{Ti}$ adhesion layer.

microscopy (TEM) images on the nanoparticles were obtained using an energy filter transmission electron microscope (LEO 922). The particles were deposited on thin carbon film coated grids and measured at $200 \mathrm{kV}$.

Preparation of Colloidal Particles. The colloidal Au and $\mathrm{Ag}$ synthesis was performed by following the procedure of $\mathrm{J}$ in et al. ${ }^{10}$ All glassware was cleaned in a bath of freshly prepared $\mathrm{HCl} / \mathrm{HNO}_{3}$ (3:1) and rinsed thoroughly in $\mathrm{H}_{2} \mathrm{O}$ prior to use. Preparation of the Au or Ag nanoparticles was performed by adding $1 \mathrm{~mL}$ of $1 \%$ (w/ v) aqueous $\mathrm{HAuCl}_{4} \cdot 3 \mathrm{H}_{2} \mathrm{O}$ or $\mathrm{AgNO}_{3}$ to $100 \mathrm{~mL}$ of ultrapure $\mathrm{H}_{2} \mathrm{O}$ under vigorous stirring, followed by the addition of $1 \mathrm{~mL}$ of $1 \%(w / v)$ aqueous sodium citrate, 1 min later. After an additional $1 \mathrm{~min}, 1 \mathrm{~mL}$ of $0.075 \%$ (w/ v) $\mathrm{NaBH}_{4}$ in $1 \%$ sodium citrate was added. The solution was stirred for $5 \mathrm{~min}$ and then stored at $4{ }^{\circ} \mathrm{C}$ until needed. The formation of the $\mathrm{Au}$ and $\mathrm{Ag}$ nanoparticles was examined using the UV-vis spectrum, which produces a strong surface plasmon band at 513 and $389 \mathrm{~nm}$ for $\mathrm{Au}$ and $\mathrm{Ag}$ nanoparticles, respectively. The size of the Au particles, verified using TEM, was found to be $4 \pm 1 \mathrm{~nm}$ (Figure 1a). The size of the Ag particles was larger and showed a larger size distribution, e.g. $21 \pm 9 \mathrm{~nm}$ (Figure 1b).

Substrate Preparation. The quartz substrates were first cleaned by immersing the samples for $1 \mathrm{~h}$ in $2 \mathrm{M} \mathrm{NaOH}$, followed by a 7 min treatment with a 1:1:5 mixture of respectively $\mathrm{H}_{2} \mathrm{O}_{2}$ $(30 \% \mathrm{v} / \mathrm{v}), \mathrm{NH}_{4} \mathrm{OH}(25 \% \mathrm{v} / \mathrm{v})$, and ultrapure $\mathrm{H}_{2} \mathrm{O}$ at $80-90{ }^{\circ} \mathrm{C}$, to achieve a freshly prepared and uniform oxide layer. The clean quartz substrates were immersed in a solution containing (3mercaptopropyl)methyltriethoxysilane dissolved in a 95:5 ( $/ \mathrm{v}$ ) methanol/ water mixture at $2 \%$ (v/ v) for up to $72 \mathrm{~h}$. Thereafter, the substrates were rinsed with methanol, dried in a $\mathrm{N}_{2}$ stream, and annealed for $10 \mathrm{~min}$ at $105^{\circ} \mathrm{C}$. Ultrathin $\mathrm{Au}$ or $\mathrm{Ag}$ particle films were prepared by immersing the mercaptosilanized samples overnight in the colloidal Au or Ag solutions. Electroless plating of gold was applied to increase the particle size after deposition of the particles on the mercaptosilanized quartz surface. The plating was performed on the basis of a procedure described by Jin et al. but using shorter deposition times. ${ }^{10}$ Briefly, the substrates were immersed in an aqueous mixture of $0.4 \mathrm{mM}$ hydroxylamine hydrochloride and a $0.1 \%(\mathrm{w} / \mathrm{v}) \mathrm{HAuCl}_{4} \cdot 3 \mathrm{H}_{2} \mathrm{O}$. The experimental details on the deposition of the self-assembled monolayers of 16-M HA on the Au and $\mathrm{Ag}$ nanoparticles coated substrates, the covalent immobilization of anti-HSA, and the conditions for the recognition of HSA have been described in Frederix et al. ${ }^{11}$

\section{RESULTS AND DISCUSSION}

The principle of biosensing via light absorption of $\mathrm{Ag}$ and $\mathrm{Au}$ nanoparticles is demonstrated using UV-vis absorbance spectroscopy (Figure 2). The sensing capabilities of $\mathrm{Ag}$ and $\mathrm{Au}$ nanoparticles thin films are compared because it is known that their plasmon characteristics are different. $6,12 \mathrm{~A}$ common problem with regard to the deposition of thin Au or Ag films of nanoparticles is related to the conventionally applied adhesion layers (e.g. $\mathrm{Cr}$ or $\mathrm{Ti}$ ), which are known to block the plasmon resonance phenomena and to broaden the plasmon resonance band. Figure 3 shows a simulation of the reflected plasmon resonance peak of

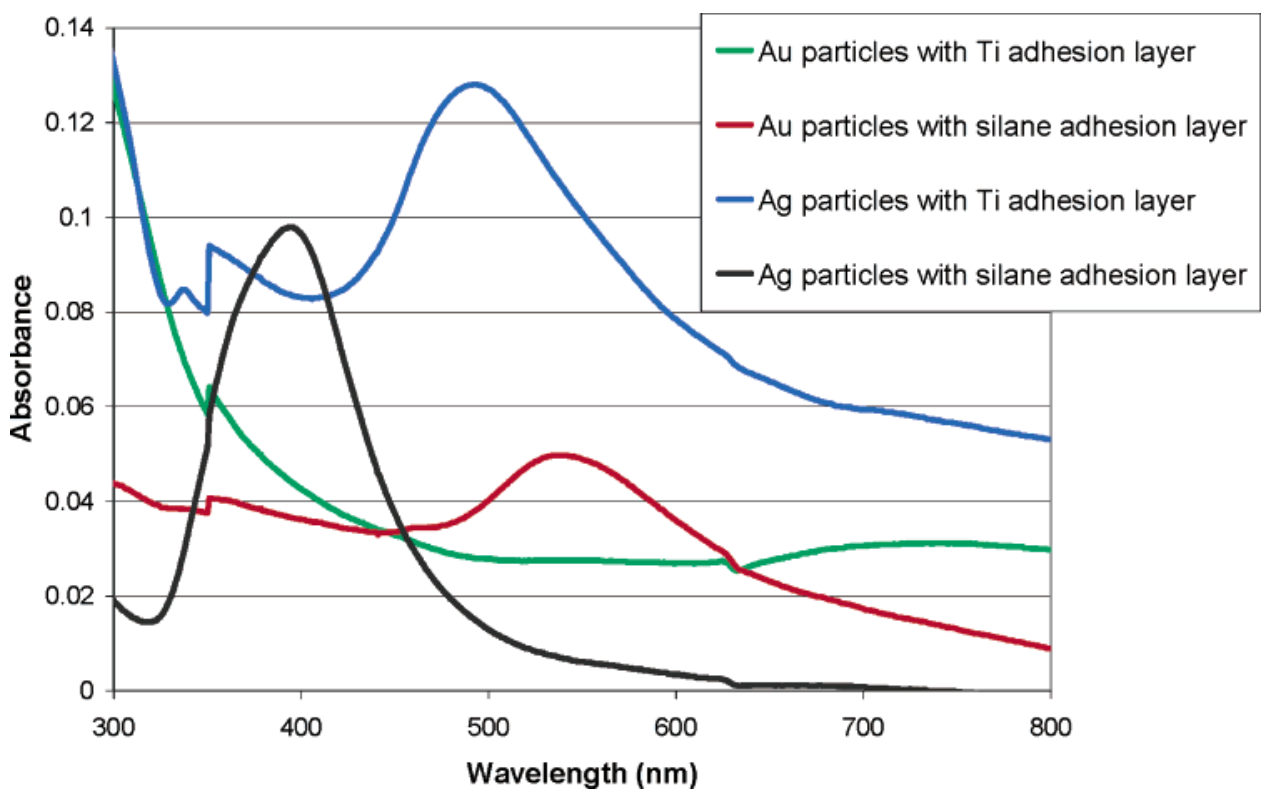

Figure 4. UV-vis absorbance spectrum comparing the use of a mercaptosilane and a Ti film as an adhesion layer for Au and Ag nanoparticles. 


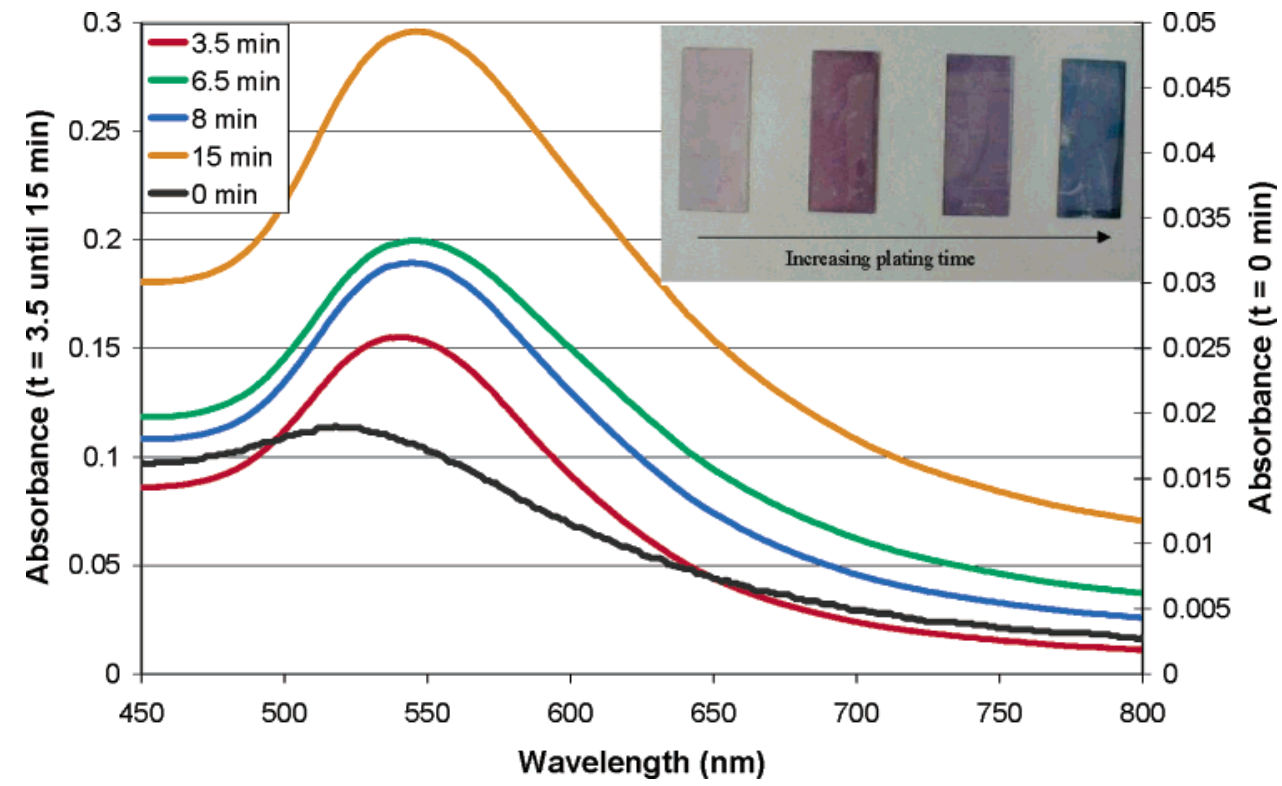

Figure 5. UV-vis absorbance spectra of mercaptosilanized quartz slides with covalently immobilized Au nanoparticles ( $t=0$ min; right $y$-axis), followed by gold electroless plating on these nanoparticles for different times ( $t=3.5$ until 15 min; left $y$-axis). The inset shows pictures of the samples.

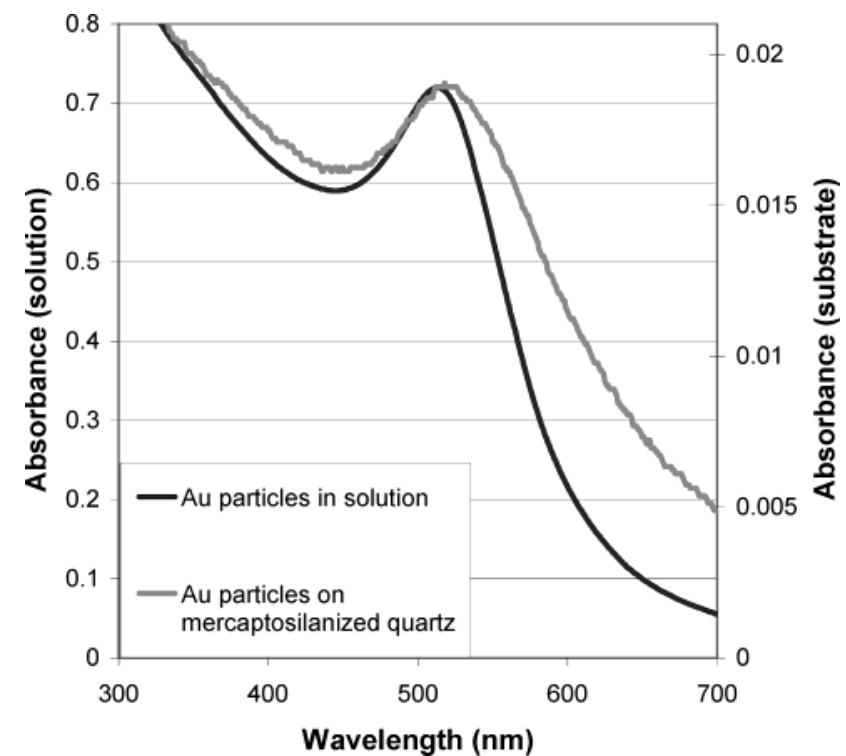

Figure 6. Absorbance spectrum of Au particles in solution (left $y$-axis) and of Au particles covalently immobilized on a mercaptosilanized quartz substrate measured in air (right $y$-axis).

a $60 \mathrm{~nm} \mathrm{Ag}$ film with and without a $\mathrm{Ti}$ adhesion layer. The simulation was performed using the optical constants (wavelength $=670 \mathrm{~nm} ; \mathrm{n}(\mathrm{Ag})=0.05+4.483 \mathrm{i} ; \mathrm{n}(\mathrm{Ti})=2.76+3.84 \mathrm{i} ; \mathrm{n}$ (water) $=1.33 ; \mathrm{n}$ (glass) $=1.518$ ) of Johnson et al. ${ }^{13}$ and the simulation method of Grossel et al. ${ }^{14}$ It is clear that when the Ti adhesion layer is present, the peak broadens and the intensity decreases, which indicates that $\mathrm{Ti}$ is not an adequate adhesion layer when

(10) Jin, Y.; Kang, X.; Song, Y.; Zhang, B.; Cheng, G.; Dong, S. Anal. Chem. 2001 $73(13), 2843$.

(11) Frederix, F.; Bonroy, K.; Laureyn, W.; Reekmans, G.; Campitelli, A.; Dehaen, W.; M aes, G. Langmuir 2003, 19, 4351.

(12) Liedberg, B.; Nylander, C.; Lundström, I. Biosens. Bioelectron. 1995, 10 (8), i.

(13) Johnson, P. B.; Christy, R. W. Phys. Rev. B 1972, 6 (12), 4370.

(14) Grossel, P.; Vigoureux, J.-M .; Baida, F. Phys. Rev. A. 1994, 50 (5), 3627.

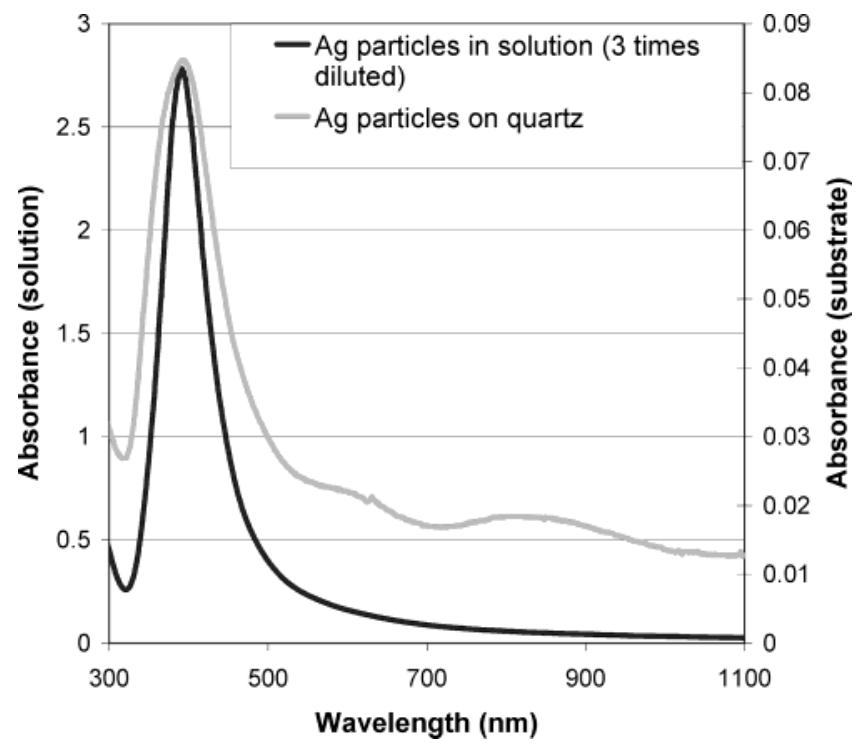

Figure 7. Absorbance spectrum of $\mathrm{Ag}$ particles in solution (left $y$-axis) and covalently immobilized on a mercaptosilanized quartz substrate measured in air (right $y$-axis).

Au and Ag nanoparticles are to be used for their optical properties. Therefore, an organic adhesion layer is used. To this aim, a mercaptosilanized surface is created on the quartz substrate, which allows coupling of the nanoparticles. Figure 4 compares the experimental absorbance spectra of the Au and Ag nanoparticles on $\mathrm{Ti}$ and on the organic adhesion layer of mercaptosilanes. Ti does not allow the adhesion of the Au particles, while it broadens and lowers the intensity of the absorbance peak of the Ag nanoparticles. M ore specifically, the peak height decreases (notice that there is an extra absorbance offset due to the Ti layer) and the full-width at half-maximum doubles.

Electroless plating of gold was applied to increase the particle size after deposition of the Au particles on the mercaptosilanized quartz surface. The gold-coated substrates change color from pink to purple to blue depending on the plating time and therefore on 
(a)
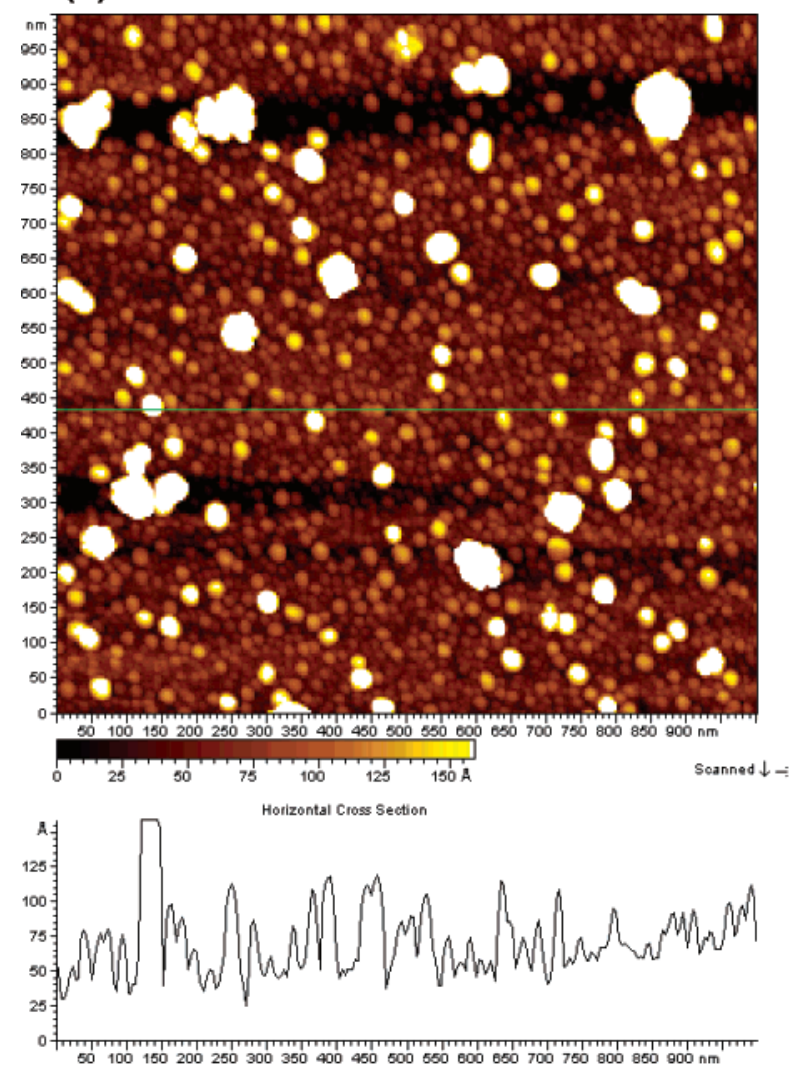

(b)
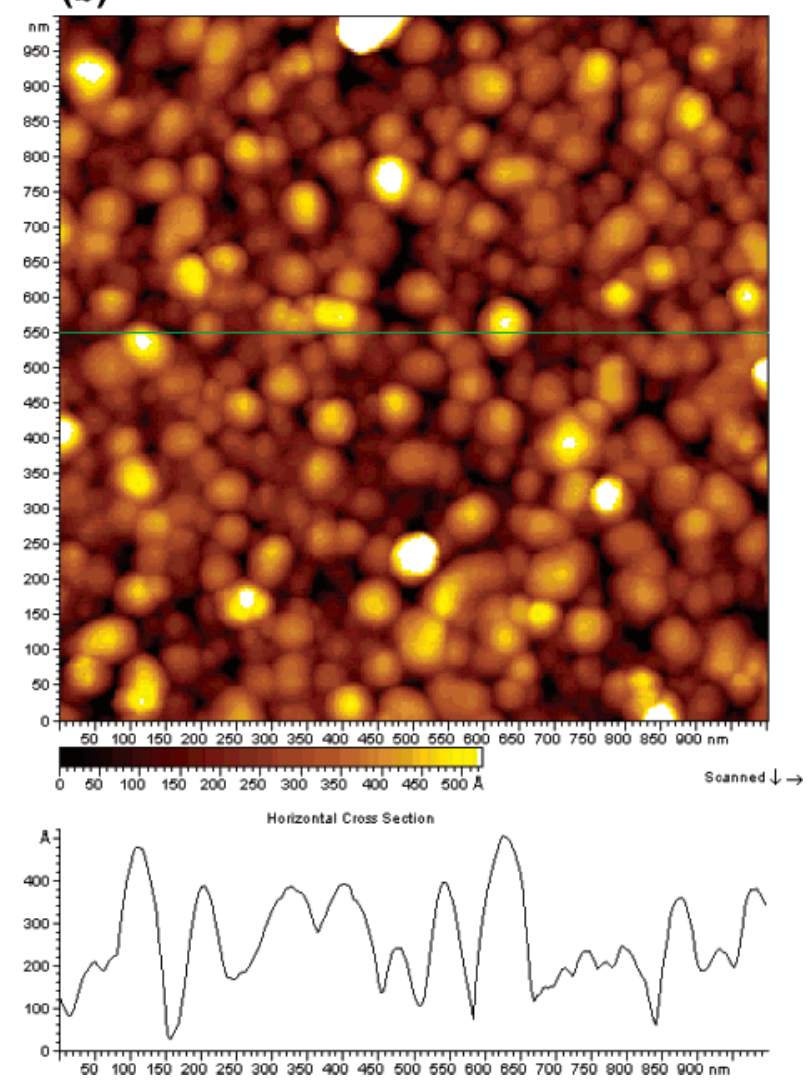

Figure 8. (a) Tapping mode atomic force microscopy image of silver particles covalently immobilized on a mercaptosilanized quartz surface. (b) Tapping mode atomic force microscopy image of gold-plated (4 min) gold particles covalently immobilized on a mercaptosilanized quartz surface.

the particle film thickness (Figure 5). The plasmon resonance absorbance maximum shows a shift of the absorbance band from $518 \mathrm{~nm}$ (bare gold particles) to $546 \mathrm{~nm}$ (15 min of Au plating) through an increased green/ red absorbance resulting in the blue/ purple color of the samples. The nanoparticles deposited on the mercaptosilanized quartz were compared with the particles in solution (Figures 6 and 7). In solution, as well as on the surface, the particle plasmon resonance absorption of the gold particles occurs at a longer wavelength than the silver particles, $\sim 513$ and $\sim 391 \mathrm{~nm}$, respectively. The starting absorbance of the unplated gold particles is approximately 4 times lower than the one of the unplated silver particles (Figures 6 and 7). Therefore, the gold particles are first plated to achieve an acceptable starting absorbance (Figure 5). Furthermore, the two kinds of particles show different behavior on the surface and in the solution. The plasmon resonance absorbance of the gold particles in solution ( $513 \mathrm{~nm}$ ) is slightly shifted to longer wavelengths (518 nm) when these particles are deposited/ aggregated on a substrate (Figure 6). The shift is depending on the size and shape of the particles and of the aggregate as well as on the interparticle distance within the aggregate. ${ }^{15}$ The intensity and the position of $\lambda_{\max }$ both reflect the extent of aggregation, resulting from coupling of surface plasmons between closely spaced particles. M etal nanoparticles embedded in glass have also been shown to introduce third-order optical nonlinearities in the composite at wavelengths close to that

(15) Hrapovic, S.; Liu, Y.; Enright, G.; Bensebaa, F.; Luong, J. H. T. Langmuir 2003, 19 (9), 3958 of the characteristic surface plasmon resonance of the embedded metal nanoparticles. ${ }^{16}$ The absorbance band not only shifts but the shape of the UV-vis spectrum also changes. Hrapovic et al. noticed similar behavior. ${ }^{15}$ From their report an absorption band around $600 \mathrm{~nm}$ is expected to originate from plasmon coupling between neighboring particles, which also shifts and broadens the plasmon band at $546 \mathrm{~nm}$. A slightly different phenomenon is noticed when the silver particles are deposited onto the surface. The plasmon resonance absorbance is not shifted, but an additional absorption band appears around $805 \mathrm{~nm}$ (Figure 7). The latter band is probably due to plasmon coupling between adjacent particles, and it is shifted much more than in the case of gold. We attribute this observation to the difference in packing between the different particles and to the size (distribution) of the silver particles on the surface ${ }^{17}$ (Figure 8 ). The size distribution of the silver particles on the surface is less uniform than the gold-plated gold particles on the surface. The gold-plated gold particles are on average larger than the silver particles, and the interparticle distance is in both systems rather small (Figure 8).

To demonstrate the biosensing ability of the silver nanoparticles, human serum albumin (HSA) was directly adsorbed onto the silver nanoparticles deposited on the surface. In Figure 9 the difference spectra, recorded in PBS, are shown. These difference

(16) Porstendorfer, J.; Berg, K.-J.; Berg, G. J. Quant. Spectrosc. Radiat. Transfer 1999, 63, 479.

(17) Haynes, C. L.; M cF arland, A. D.; Zhao, L.; Van Duyne, R. P.; Schatz, G. C.; Gunnarsson, L.; Prikulis, J.; Kasemo, B.; Käll, M. J. Phys. Chem. B 2003 ASAP article. 


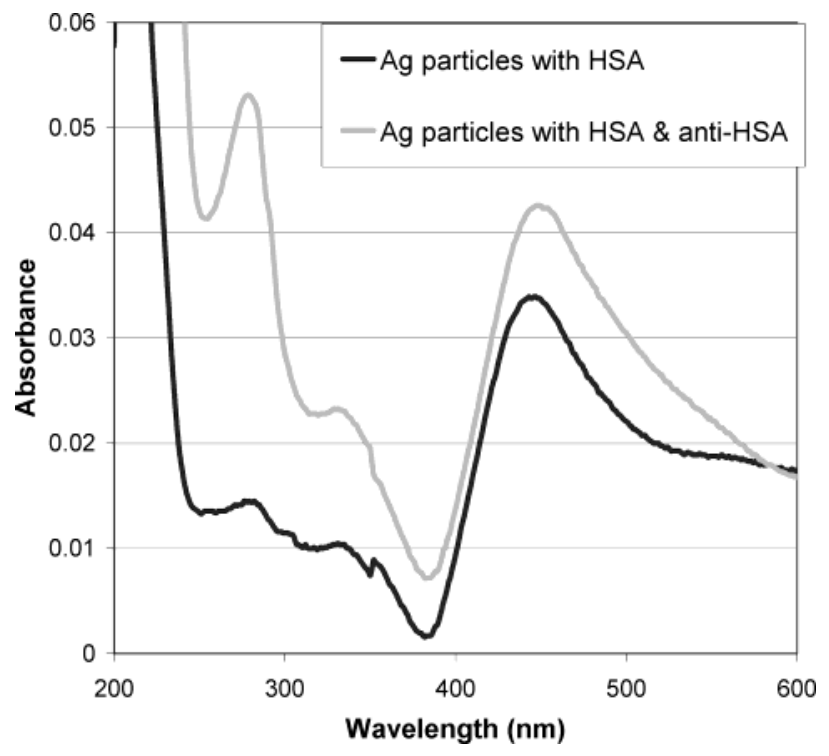

Figure 9. Difference absorbance spectra for HSA immobilized on $\mathrm{Ag}$ particles and the subsequent recognition of anti-HSA by the immobilized HSA.

spectra represent the absorbance spectra, with bare quartz as a background, corrected for the absorbance spectra of the bare particle film. The adsorption of HSA on the deposited silver particles was performed by immersion of the substrate in a solution of $1.244 \mathrm{mg} / \mathrm{mL}$ HSA in PBS for $120 \mathrm{~min}$, followed by thoroughly rinsing with PBS and measuring in PBS. After adsorption, an enhancement of the particle plasmon absorption at a wavelength of $\sim 443 \mathrm{~nm}$ is observed from the difference spectrum. Kalyuzhny et al. also noticed a similar phenomenon for thiols on gold particles..$^{18} \mathrm{In}$ addition, the interband absorption at short wavelengths ( $280 \mathrm{~nm}$ ) increases, which can only be observed when the particles are deposited on a substrate transparent at these wavelengths (quartz in this case). Second, the HSA immobilized sample was immersed in a solution of the complementary antibody ( $140 \mu \mathrm{g} / \mathrm{mL}$ anti-HSA in PBS) for 180 min using an identical rinsing procedure. The plasmon absorbance was found to change and shift to higher wavelength after the affinity binding. More specifically, the maximum in the difference absorbance spectrum shifts from 443 to $447 \mathrm{~nm}$ and increases by one-quarter of its initial value upon anti-HSA recognition. This indicates that the molecular recognition of HSA and anti-HSA can be detected as a change in the absorption probability and/ or a change in the resonance wavelength. In addition, again a strong interband absorption is observed at $\sim 280 \mathrm{~nm}$, which we relate to a transition in the aromatic amino acids (phenylalanine, tyrosine, and tryptophan) of the proteins. This 2.7 times increase upon anti-HSA recognition is a consequence of the large change in the refractive index at resonance of the amino acids, which increases the absorption probability of the nanoparticles. It should be noticed that there is no absorption band for a bare quartz slide, which is coated with proteins, since the absorption intensity is too weak to be detected. Therefore, we assign this novel effect observed for the coated particles to a resonant absorption enhancement by the particles.

(18) Kalyuzhny, G.; Vaskevich, A.; Ashkenasy, G.; Shanzer, A.; Rubinstein, I. J. Phys. Chem. B 2000, 104, 8238.

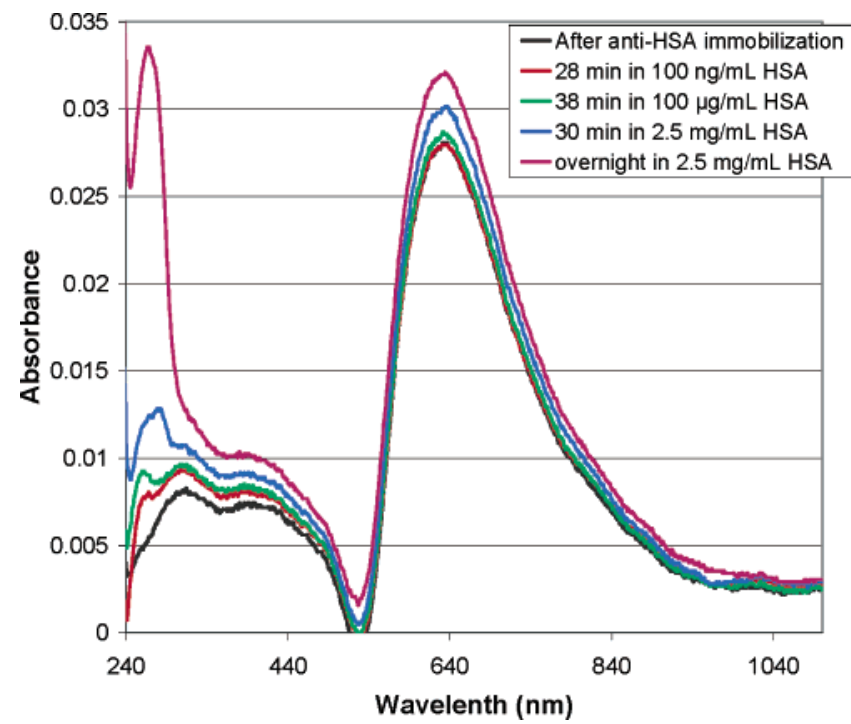

Figure 10. Difference absorbance spectra of anti-HSA immobilized on gold-plated gold particles and the recognition of HSA by the immobilized anti-HSA.

\begin{tabular}{|c|c|c|c|c|}
\hline & $\begin{array}{l}\text { increas } \\
\text { comparec } \\
\text { of the pre } \\
\text { on of } \mathrm{H}\end{array}$ & $\begin{array}{l}\text { of abs } \\
\text { to the abs } \\
\text { ous concn } \\
\text { A (in } \% \text { ) }\end{array}$ & $\begin{array}{l}\text { increa } \\
\text { compare } \\
\text { after } \\
\text { immobili }\end{array}$ & $\begin{array}{l}\text { of abs } \\
\text { o the abs } \\
\text { i-HSA } \\
\text { ion (in \% }\end{array}$ \\
\hline & $634 \mathrm{~nm}$ & $278 \mathrm{~nm}$ & $634 \mathrm{~nm}$ & $278 \mathrm{~nm}$ \\
\hline $\begin{array}{l}28 \mathrm{~min} \text { in } 100 \mathrm{ng} / \mathrm{mL} \text { HSA } \\
38 \mathrm{~min} \text { in } 100 \mu \mathrm{g} / \mathrm{mL} \text { HSA } \\
30 \mathrm{~min} \text { in } 2.5 \mathrm{mg} / \mathrm{mL} \text { HSA } \\
\text { overnight in } 2.5 \mathrm{mg} / \mathrm{mL} \mathrm{HSA}\end{array}$ & $\begin{array}{l}0 \\
2.2 \\
5.6 \\
6.5\end{array}$ & $\begin{array}{r}27.4 \\
9.2 \\
47.9 \\
146.7\end{array}$ & $\begin{array}{r}0 \\
1.7 \\
7.4 \\
14.3\end{array}$ & $\begin{array}{r}27.4 \\
39.2 \\
105.9 \\
407.8\end{array}$ \\
\hline
\end{tabular}

Finally, immunosensing experiments were performed on a thin layer of plated gold ( 4 min of plating). These experiments clearly show the potential of this sensing method for real biosensor applications (Figure 10; difference spectra). A self-assembled monolayer (SAM) of 16-M HA was deposited on the gold particle film. Subsequently, a monolayer of anti-HSA was covalently immobilized by a procedure described elsewhere. ${ }^{11}$ As shown in Figure 10, the molecular recognition of anti-HSA and HSA can again be detected as a change in the plasmon absorption probability and a change in the resonance frequency (at wavelength $\sim 634 \mathrm{~nm}$ ). At $634 \mathrm{~nm}$, a subsequent increase is noticed of $0 \%$ after $100 \mathrm{ng} / \mathrm{mL}$ HSA for $28 \mathrm{~min}$, of 2.2\%after $100 \mu \mathrm{g} / \mathrm{mL} \mathrm{HSA}$ for $38 \mathrm{~min}$, of $5.6 \%$ after $2.5 \mathrm{mg} / \mathrm{mL}$ HSA for $30 \mathrm{~min}$, and of $6.5 \%$ after $2.5 \mathrm{mg} / \mathrm{mL}$ HSA overnight (Table 1). No increase was noticed when a nonspecific analyte, e.g. 10 and $100 \mu \mathrm{g} / \mathrm{mL}$ IgG for $30 \mathrm{~min}$, was sent over the surface. The nonspecific analyte was sent over the immobilized anti-HSA before HSA was sent over it. The increase in absorbance compared to the signal after antiHSA immobilization is also shown in Table 1. A concentration of $100 \mathrm{ng} / \mathrm{mL}$ HSA for 28 min seems to be not detectable using this kind of setup. We should state that the current setup does not allow stirring of the solution and therefore induces mass transport limitations. The bulk interband absorption (between 240 and 320 $\mathrm{nm}$ ) also increases for reasons stated above. Table 1 also lists the subsequent increase after injecting different concentrations of HSA and the total increase compared to the absorbance signal 
after anti-HSA immobilization. The absorbance enhancement of the characteristic band at $\sim 278 \mathrm{~nm}$ is found to be very strong and can be even more sensitive than plasmon band changes.

\section{CONCLUSION}

In this study an organic adhesion layer to immobilize gold and silver nanoparticles onto quartz substrates was used and these nanoparticles were functionalized with bioreceptors for biosensing applications. Upon binding of proteins to the gold and silver particles, changes in both the intensity and the wavelength of the particle resonances and the intensity of interband absorptions are observed. In addition, a resonant enhancement of the interband absorption was found to occur. To our knowledge this is the first report of biosensing on quartz substrates in combination with silver and gold particles, based on a resonant enhancement of the interband absorption bands. The method presented is versatile, allowing applications in the liquid and gas phases. Furthermore, this novel approach is promising as an easy and cost-effective alternative for conventional biosensing techniques. Future work will focus on the sensitivity and the quantitative interpretation of the intensity increases upon binding to different kind of particles.

\section{ACKNOWLEDGMENT}

The authors thank the IWT (Instituut voor de aanmoediging van Innovatie door Wetenschap en Technologie in Vlaanderen) for its financial support. We also thank Olivier Richard from IM EC and Etienne Ferain of L'Université Catholique de Louvain (Unité de Physique et de Chimie des Hauts Polymères) for performing the TEM measurements.

Received for review June 18, 2003. Accepted September 17, 2003.

AC0346609 